\title{
A Tiling Lemma and Its Application to the Ratio Test for Convergence of Series
}

\author{
James D. Stein Jr., Linda Ho \\ Department of Mathematics California State University Long Beach, CA \\ Department of Mathematics El Camino College Torrance, CA \\ E-mail:jimstein@csulb.edu,lho@elcamino.edu \\ Received July 5, 2011; revised August 1, 2011; accepted August 12, 2011
}

\begin{abstract}
We prove that any collection which tiles the positive integers must contain one of two types of sub-collections. We then use this result to prove a variation of the Ratio Test for convergence of series. This version of the Ratio Test shows the convergence of certain series for which the Root Test (which is known to be more powerful than the conventional Ratio Test) fails. This version of the Ratio Test is also used to prove a version of the Banach Contraction Principle for self-maps of a complete metric space.
\end{abstract}

Keywords: Tiling, Ratio Test, Banach Fixed-Point Theorem

\section{Introduction}

It is shown in [1] that tiling results can be used in some fixed-point theorems to circumvent tedious and computational analytic arguments. We show in this paper that tiling results can also be used to improve the Ratio Test for convergence of series. The classical Ratio Test is known to be dominated by the Root Test; if the Ratio Test demonstrates that a series converges, the Root Test would also yield this result. The improved Ratio Test presented in this paper can be used to demonstrate the convergence of some series for which the Root Test will not yield conclusive results.

\section{A Simple Tiling Result}

On the real line, let $j \leq k$, and let $t[j, k]$ denote the tile extending from $j-1 / 2$ to $k+1 / 2$; that is, the closed interval $[j-1 / 2, k+1 / 2]$. Since we are only concerned with integers in this paper, notice that this tile covers all the integers from $\mathrm{j}$ through $k$ inclusive.

Lemma 2.1 Let $T$ be a collection of distinct tiles of the above form such that every positive integer is covered by at least one tile in $T$. Then either

1) $\exists N$ such that sup $\{k: t[N, K] \in T\}=+\infty$, or

2) there is a sub-collection $T^{*}$ of $T$, $T^{*}=\left\{T\left[\alpha_{n}, \beta_{n}\right]: n=1,2, \cdots\right\}$ such that for each $n$, $\alpha_{n+1}>\alpha_{n}, \beta_{n+1}>\beta_{n}$, and each positive integer belongs to either one or two tiles in $T^{*}$.
Proof: Suppose that 1) does not hold. We can therefore assume that, for each $n, \exists$ only finitely many pairs $(p, q)$ with $p \leq n \leq q$ and $t[p, q] \in T$. Let $q=\max \{k: t[1, k] \in T\}$, and let $\alpha_{1}=1, \beta_{1}=q$.

Having chosen $\alpha_{n}$ and $\beta_{n}$ such that all positive integers $\leq \beta_{n}$ belong to one of the tiles $t\left[\alpha_{1}, \beta_{1}\right], \cdots$, $t\left[\alpha_{n}, \beta_{n}\right]$, let $\beta_{n+1}=\max \left\{k: \exists j, k\right.$ with $j \leq \beta_{n}+1 \leq k$ and $t[j, k] \in T\}$. Let $\alpha_{n+1}=\max \left\{j: j \leq \beta_{n+1}\right.$ and $t\left[j, \beta_{n+1}\right]$ $\in T\}$. Note that

1) $\beta_{n+1}>\beta_{n}$ by construction, and all positive integers $\leq \beta_{n+1}$ belong to one of the tiles $t\left[\alpha_{1}, \beta_{1}\right], \cdots, t\left[\alpha_{n+1}, \beta_{n+1}\right]$

2) $\alpha_{n+1}>\alpha_{n}$ : if $\alpha_{n+1} \leq \alpha_{n}$, then we should have chosen $\beta_{n+1}$ instead of $\beta_{n}$, since $t\left[\alpha_{n}, \beta_{n}\right]$ is a proper subset of $t\left[\alpha_{n+1}, \beta_{n+1}\right]$, contradicting the maximality of $\beta_{n}$.

Notice that no three consecutive tiles of the form $t\left[\alpha_{n}\right.$, $\left.\beta_{n}\right]$ overlap. If $k \in t\left[\alpha_{n+j}, \beta_{n+j}\right]$ for $j=0,1,2$, then $\alpha_{n+j} \leq k$ $\leq \beta_{n+j}$ for $j=0,1,2$. But then $k \leq \beta_{n}<\beta_{n}+1$ and $\beta_{n+2}>\beta_{n+1}$ $\Rightarrow \beta_{n}+1 \in t\left[\alpha_{n+2}, \beta_{n+2}\right]$, contradicting the maximality of $\beta_{n+1}$. Therefore each integer $\mathrm{k}$ either belongs to a unique tile $t\left[\alpha_{n}, \beta_{n}\right]$ or to two consecutive tiles: $t\left[\alpha_{n}, \beta_{n}\right]$ and $t\left[\alpha_{n+1}, \beta_{n+1}\right]$.

The collection $T^{*}=\left\{t\left[\alpha_{n}, \alpha_{n}\right]: n=1,2, \cdots\right\}$.

\section{An Application of Lemma 2.1 to the Ratio Test}

We now use Lemma 2.1 to prove our version of the Ratio Test.

Theorem 3.1 (Ratio Test)-Assume that $a_{1} \geq a_{2}$ 
$\geq \cdots \geq 0$ and $\lim a_{n}=0$. Assume further that $\exists M \in$ $(0,1)$ such that, for every integer $n$, there exist integers $j$, $k$ with $j \leq n \leq k$ and $a_{j+1}+\cdots+a_{k+1} \leq M\left(a_{j}+\cdots+a_{k}\right)$. Then $\sum_{n=1}^{\infty} a_{n}$ converges.

Proof: Suppose that, for $n>1, b_{2}+\cdots+b_{n+1} \leq M\left(b_{1}\right.$ $\left.+\cdots+b_{n}\right)$.

Then $b_{2}+\cdots+b_{n}+b_{n+1} \leq M b_{1}+M\left(b_{2} \cdots+b_{n}\right) \Rightarrow(1-$ M) $\left(b_{2} \cdots+b_{n}\right) \leq M b_{1}-b_{n+1}$; adding $(1-M) b_{1}$ to both sides and dividing by $(1-M)$ yields $b_{1}+\cdots+b_{n} \leq$ $\frac{1}{1-M}\left(b_{1}-b_{n+1}\right)$.

This inequality also holds if $n=1$, for then $b_{2} \leq M b_{1}$ $\Rightarrow b_{2} \leq M b_{1}+b_{1}-b_{1} \Rightarrow b_{2} \leq b_{1}+(M-1) b_{1} \Rightarrow(1-M)$ $b_{1} \leq b_{1}-b_{2} \Rightarrow b_{1} \leq \frac{1}{1-M}\left(b_{1}-b_{2}\right)$.

If $1<j \leq k$, we say that the tile $t[j, k]$ belongs to the collection $T$ if $a_{j}+\cdots+a_{k} \leq M\left(a_{j-1}+\cdots+a_{k-1}\right) \leq \frac{1}{1-M}$ $\left(a_{j-1}-a_{k}\right)$.

The hypothesis enables us to apply the Lemma, and we can conclude that either

1) $\exists N$ such that sup $\{k: t[N, k] \in T\}=+\infty$, or

2) there is a sub-collection $T^{*}$ of $T$ of the type described in the Lemma.

If (1) holds, we can assume WLOG that $N=2$, and so $\exists$ an increasing sequence of integers $\left\{q_{n}: 1,2, \cdots\right\}$ with $1<q_{1}$ and $a_{2}+\cdots+a_{q_{n}+1} \leq M\left(a_{1}+\cdots+a_{q_{n}}\right)$. Therefore $a_{1}+\cdots+a_{q_{n}} \leq \frac{1}{1-M}\left(a_{1}+\cdots+a_{q_{n}+1}\right) \leq \frac{1}{1-M} a_{1}$, so the partial sums of $\sum_{j=1}^{\infty} a_{j}$ form a bounded monotone sequence.

We can therefore assume from the Lemma that we have a sub-collection $T^{*}$ of $T$ consisting of tiles $t\left[p_{n}, q_{n}\right]$ such that each integer belongs to either one or two tiles in $T^{*}$, and such that $p_{n}<p_{n+1}$ and $q_{n}<q_{n+1}$ for each integer $n$, so if an integer belongs to two tiles in $T^{*}$, they are consecutive tiles.

Observe that $\sum_{n=1}^{\infty} a_{n} \leq \sum_{n=1}^{\infty} \sum_{k=p_{n}}^{a_{n}} a_{k}$. We can subdivide the right-hand side summation over the tiles $t\left[p_{n}, q_{n}\right]$ into three parts: tiles of length 1 , tiles of length longer than 1 which do not overlap other tiles in $T^{*}$, and tiles of length longer than 1 which overlap other tiles in $T^{*}$.

The summation over tiles of length $1\left(q_{n}=p_{n}\right)$ : if there are only a finite number of such tiles, the sum of these terms is clearly finite. If there are infinitely many such terms, since the sequence $\left\{a_{n}: n=1,2, \cdots\right\}$ is decreasing, the summation over all such terms is dominated by the geometric series whose first term is $a_{1}$ and whose ratio is
$M$.

Once the tiles of length 1 have been eliminated, we can renumber the remaining tiles as $\left\{t\left[p_{n}, q_{n}\right]: n=1,2, \cdots\right\}$ with $p_{n}<p_{n+1}$ and $q_{n}<q_{n+1}$. We look at two types of blocks consisting of consecutive tiles $t\left[p_{k}, q_{k}\right], t\left[p_{k+1}\right.$, $\left.q_{k+1}\right], \cdots, t\left[p_{n}, q_{n}\right]$. In the first type of block, consecutive tiles do not overlap; in the second, consecutive tiles overlap. The remaining tiles can be divided into alternating blocks of these two types simply by continuing to examine successive tiles to see whether they overlap or not.

There are two cases: either there are infinitely many blocks of each of the two types, or there are only finitely many blocks of each of the two types; this last case occurs when all but finitely many tiles belong to a single block. We examine the first case, and then discuss how the analysis presented therein also handles the second case.

If consecutive tiles $t\left[p_{k}, q_{k}\right], t\left[p_{k+1}, q_{k+1}\right], \cdots, t\left[p_{n}, q_{n}\right]$ do not overlap, then $p_{k}<q_{k}<p_{k+1}<q_{k+1}<\cdots<p_{n}<q_{n}$. The inequality $p_{j}<q_{j}$ is strict because the tiles of length 1 were treated in the discussion above, and the inequality $q_{j}<p_{j+1}$ is strict because consecutive tiles do not overlap. Then

$$
\sum_{j=1}^{n} \sum_{k=p_{j}}^{q_{j}} a_{k} \leq \frac{1}{1-M}\left[\left(a_{p_{k}-1}-a_{q_{k}}\right)+\cdots+\left(a_{p_{n}-1}-a_{q_{n}}\right)\right] .
$$

This sum dominates the contribution from a typical block of consecutive non-overlapping tiles. Since $p_{k}<q_{k}$ $<p_{k+1}<q_{k+1}<\cdots<p_{n}<q_{n}$, the terms in the above sum decrease within a particular block, and since there is a gap between this block and the next block of consecutive non-overlapping tiles, the last term from the above sum is greater than the first term in the corresponding sum from the next block. Adding up the dominating sums from all such blocks and using the Alternating Series Test results in a convergent series.

Finally, assume that consecutive tiles $t\left[p_{k}, q_{k}\right], t\left[p_{k+1}\right.$, $\left.q_{k+1}\right], \cdots, t\left[p_{n}, q_{n}\right]$ overlap. Because $t\left[p_{j}, q_{j}\right]$ and $t\left[p_{j+1}, q_{j+1}\right]$ overlap, $q_{j}$ is common to both tiles, so $p_{j}<p_{j+1} \leq q_{j}$. No integer belongs to three tiles, so $q_{j}$ does not belong to $t\left[p_{j+2}, q_{j+2}\right]$, and so $q_{j}<p_{j+2}$. Therefore $p_{k}<p_{k+1} \leq q_{k}<$ $p_{k+2} \leq q_{k+1}<\cdots \leq q_{n-2}<p_{n} \leq q_{n-1}<q_{n}$. We use the same initial estimate as in the previous case, but permute the final dominating sum slightly.

$$
\begin{aligned}
& \sum_{j=1}^{n} \sum_{k=p_{j}}^{q_{j}} a_{k} \leq \frac{1}{1-M}\left[\left(a_{p_{k}-1}-a_{q_{k}}\right)+\cdots+\left(a_{p_{n}-1}-a_{q_{n}}\right)\right] \\
& =\frac{1}{1-M}\left\{\left(a_{p_{k}-1}-a_{q_{n}}\right)\right. \\
& \left.+\left[\left(a_{p_{k}}-a_{q_{k}}\right)+\cdots+\left(a_{p_{n}-1}-a_{q_{n}-1}\right)\right]\right\}
\end{aligned}
$$

The sum in brackets consists of two components: the 
first term $\left(a_{p_{k}-1}-a_{q_{n}}\right)$ and the sum $\left[\left(a_{p k}-a_{q k}\right)+\cdots+\right.$ $\left.\left(a p_{n-1}-a q_{n-1}\right)\right]$. If we add up the first terms from all blocks of consecutive overlapping tiles, the sum converges by the Alternating Series Test, and the same can be said of all the bracketed sums of the form $\left[\left(a_{p k}-a_{q k}\right)\right.$ $\left.+\cdots+\left(a p_{n-1}-a q_{n-1}\right)\right]$. This completes the proof in the case where there are infinitely many blocks of each of the two types.

If all but finitely many of the tiles belong to a single block, the dominating sum is a sum over infinitely many tiles. The identical analysis (with upper limit of $\infty$ instead of $n$ ) shows that the dominating sum is an alternating series which converges by the Alternating Series Test.

The classical Ratio Test requires that the ratio of successive terms be dominated by a constant $<1$. In the Ratio Test presented in Theorem 1, the information is not necessarily obtainable about individual terms, but about blocks of consecutive terms. As a result, this ratio test might be useful in situations when the individual terms of the series are not known, but information about blocks of consecutive terms is available.

\section{Necessity of the Contractivity Hypothesis}

In the classical Ratio Test, it is not required that successive terms decrease, although that is a trivial consequence of the hypothesis that the ratio of successive terms be less than 1 . The Root Test does not require that successive terms decrease, so one might wonder if this hypothesis is really needed in Theorem 1 . The following example addresses this question.

Example 4.1. Let $M=0.9$. For $n=0,1,2, \cdots$ define

$$
\begin{aligned}
& a_{3 n+1}=1 / n \quad a_{3 n+2}=\frac{1}{10 n} \quad a_{3 n+3}=\frac{1}{10 n} \\
& \text { Let } p_{n}=3 n+1 \quad \text { and } q_{n}=3 n+4 \text { for } n=0,1,2, \cdots
\end{aligned}
$$

Notice that each integer belongs to at least one and at most two intervals $\left[p_{n}, q_{n}\right]$.

We want to ensure that

$a_{p_{n}+1}+\cdots+a_{q_{n}+1} \leq M\left(a_{p_{n}}+\cdots+a_{q_{n}}\right)$. To show this,

notice that

$$
\begin{aligned}
\frac{1}{10 n}+\frac{1}{10 n}+\frac{1}{n+1}+\frac{1}{10(n+1)} & =\frac{2}{10 n}+\frac{11}{10(n+1)} \\
& =\frac{13 n+2}{10 n(n+1)} \\
& =\frac{130 n+20}{10 n(n+1)}
\end{aligned}
$$

So $a_{p_{n}+1}+\cdots+a_{q_{n}+1}=\frac{130 n+20}{100 n(n+1)}$.

Also

$$
\begin{aligned}
0.9\left(\frac{1}{n}+\frac{1}{10 n}+\frac{1}{10 n}+\frac{1}{n+1}\right) & =\left(\frac{9}{10}\right)\left(\frac{12}{10 n}+\frac{1}{n+1}\right) \\
& =\left(\frac{9}{10}\right)\left(\frac{22 n+12}{10 n(n+1)}\right) \\
& =\frac{198 n+108}{100 n(n+1)}
\end{aligned}
$$

So $M\left(a_{p_{n}}+\cdots+a_{q_{n}}\right)=\frac{198 n+108}{100 n(n+1)}$.

Therefore, $a_{p_{n}+1}+\cdots+a_{q_{n}+1} \leq M\left(a_{p_{n}}+\cdots+a_{q_{n}}\right)$.

Since $\lim _{n \rightarrow \infty} a_{3 n+1}=\lim _{n \rightarrow \infty} a_{3 n+2}=\lim _{n \rightarrow \infty} a_{3 n+3}=0$, so

$\lim _{n \rightarrow \infty} a_{n}=0$.

Note also that $\sum_{n=1}^{\infty} a_{n}$ diverges, since it dominates the

\section{An Example for Which Theorem 3.1 Succeeds Where the Root Test Fails}

It is well known that, although the Ratio Test is easier to apply than the Root Test, the Root Test is more discriminating (see [2], Theorem 3.37). We now present a series for which the Ratio Test of Theorem 3.1 demonstrates convergence, but for which the Root Test is inconclusive.

Example 5.1 The basis of our example is the following: suppose $M \in(0,1), a_{1}$ and an integer $n$ are given, and $a_{2}=\cdots=a_{n+1} \leq\left[\frac{M}{(1-M) n+M}\right] a_{1}$

Then $((1-M) n+M) a_{n+1} \leq M a_{1}$.

$$
\begin{aligned}
& \Rightarrow n a_{n+1} \leq M\left(a_{1}+(n-1) a_{n+1}\right) \\
& \Rightarrow a_{2}+\cdots+a_{n+1} \leq M\left(a_{1}+\cdots+a_{n}\right) .
\end{aligned}
$$

Notice that $(1-M) n+M=n-(n-1) M>1$, and so $a_{1} \geq \cdots \geq a_{n} \geq a_{n+1}$.

Now let $b, c>0$, and consider $u=\lim _{x \rightarrow \infty}\left(\frac{b}{c x+d}\right)^{\frac{1}{x}}$. Taking natural logarithms of both sides yields $\ln u=\lim _{x \rightarrow \infty} \frac{1}{x} \ln \left(\frac{b}{c x+d}\right)=\lim _{x \rightarrow \infty} \frac{\ln b-\ln (c x+d)}{x}$, and applying L'Hopital's Rule we obtain $\ln u=\lim _{x \rightarrow \infty} \frac{\frac{-c}{c x+d}}{1}=0$, and so $u=1$.

Consequently we see that for any $\delta>0$, we can always find an integer $N$ such that $n \geq N \Rightarrow$

$$
\left[\frac{M a_{1}}{(1-M) n+M}\right]^{\frac{1}{n}}>1-\delta .
$$


We use this to construct a series $\sum_{n=1}^{\infty} b_{n}$ of decreasing terms which satisfies the hypotheses of the Theorem, but for which the Root Test is inconclusive.

Let $b_{1}=1$, let $a_{1}=b_{1}$, and choose an integer $N_{1}$ such that $n \geq N_{1} \Rightarrow\left[\frac{M a_{1}}{(1-M) n+M}\right]^{\frac{1}{n}}>1-\frac{1}{2}$ and $\frac{M a_{1}}{(1-M) n+M}<1$.

Let $b_{2}=\cdots=b_{N_{1+1}}=\frac{M a_{1}}{(1-M) N_{1}+M}$.

Having defined $b_{1}=\cdots=b_{N_{p+1}}$, let $a_{1}=b_{N_{p+1}}$ and choose an integer $N_{p+1}>N_{p}$ such that $n \geq N_{p+1} \Rightarrow$ $\left[\frac{M a_{1}}{(1-M) n+M}\right]^{\frac{1}{n}}>1-\frac{1}{p+2}$ and $\frac{M a_{1}}{(1-M) n+M}<1$.

Let $b_{N_{p+2}}=\cdots=b_{N_{p+1}}+1=\frac{M a_{1}}{(1-M) N_{p+1}+M}$. As constructed, the sequence $\left\{b_{n}: n=1,2, \cdots\right\}$ satisfies the hypotheses of the theorem, and so $\sum_{n=1}^{\infty} b_{n}$ converges.

However, by construction we also have $\lim \sup \sqrt[n]{b_{n}}=1$, and so the Root Test is inconclusive for this series.

\section{Applications}

Our first application is that the tiling result of Lemma 2.1 also applies to the Comparison Test for convergence of series.

Let $\sum_{n=1}^{\infty} b_{n}$ be a convergent series of positive terms. Let $\sum_{n=1}^{\infty} a_{n}$ be a series of positive terms. If $k \leq n$, let $C[k, n]$ be the statement: $a_{k}+\cdots+a_{n} \leq b_{k}+\cdots+b_{n}$. We will also let $[p, q]=\{k$ : $k$ is an integer, $p \leq k \leq q\}$.

Theorem 6.1 Suppose $\exists N$ э $n \geq N \Rightarrow \exists p$, $q$ with $p \leq$ $n \leq q$ and $C[p, q]$. Then $\sum_{n=1}^{\infty} a_{n}$ converges.

Proof: We can assume WLOG that $N=1$. If $\exists$ an integer $p$ э sup $\{q: C[p, q]\}=+\infty$, then $\sum_{n=1}^{\infty} a_{n}$ converges because if $C\left[p, q_{k}\right]$ for $q_{1}<q_{2}<\cdots$, then the partial sums $\sum_{j=1}^{a_{k}} a_{j}$ form a bounded monotone increasing sequence.

We can therefore assume that, for each $n, \exists$ only finitely many pairs $(p, q)$ with $p \leq n \leq q$ and $C[p, q]$. Applying the Lemma, we can find a sequence of tiles $\left\{t\left[\alpha_{n}, \beta_{n}\right]: n=1,2, \cdots\right\}$ such that $C\left[\alpha_{n}, \beta_{n}\right]$ for each $n$ and each integer $k$ either belongs to a unique interval $\left[\alpha_{n}, \beta_{n}\right]$ or to two consecutive intervals: $\left[\alpha_{n}, \beta_{n}\right]$ and $\left[\alpha_{n+1}, \beta_{n+1}\right]$.

Note that, for any $n$,

$$
\sum_{j=1}^{\beta_{n}} a_{j} \leq \sum_{j=1}^{n} \sum_{k=\alpha_{j}}^{\beta_{j}} a_{k} \leq \sum_{j=1}^{n} \sum_{k=\alpha_{j}}^{\beta_{j}} b_{k} \leq 2 \sum_{j=1}^{\beta_{n}} b_{j} \leq 2 \sum_{j=1}^{\infty} b_{j}
$$

since each $k$ appears in a maximum of two $\left[\alpha_{j}, \beta_{j}\right]$. So the partial sums of $\sum_{j=1}^{\infty} a_{j}$ form a bounded monotone sequence, and so $\sum_{j=1}^{\infty} a_{j}$ converges.

The second application uses Theorem 3.1 to obtain a different version of the Banach Contraction Principle. As mentioned earlier, [1] shows the use of tiling arguments in fixed-point theorems; Theorem 6.2 represents another such application. There may well be similar applications to other fixed-point theorems than the one presented here, especially in light of the fact that convergent series are frequently used in demonstrating the existence of fixed points.

Theorem 6.2 Let $(X, d)$ be a complete metric space, and assume that $T$ is a self-map of $X$ satisfying $d(T x, T y)$ $\leq d(x, y)$. Suppose there is an $M \in(0,1)$ such that for each pair $x, y \in X$, there is a positive integer $n=n(x, y)$ such that $d(T x, T y)+\cdots+d\left(T^{n+1} x, T^{n+1} y\right) \leq M[d(x, y)$ $\left.+\cdots+d\left(T^{n} x, T^{n} y\right)\right]$. Then $T$ has a unique fixed point.

Proof: The proof of this corollary uses standard ideas, and will be abbreviated. Start with the pair $(x, T x)$, and use the hypothesis iteratively to obtain a convergent (by Theorem 1) series $\sum_{n=1}^{\infty} d\left(T^{n} x, T^{n+1} x\right)$. The convergence of this series implies that the sequence of iterates $\left\{T^{n} x: \mathrm{n}=\right.$ $1,2, \cdots\}$ is Cauchy, and its limit can be shown by the usual methods to be a fixed point of $T$. Note that the contractivity hypothesis $d(T x, T y) \leq d(x, y)$ is needed to satisfy the requirement that the terms of the series decrease.

To show that the fixed point is unique, suppose that $x$ and $y$ are two distinct fixed points. Let $n$ be an integer such that $d(T x, T y)+\cdots+d\left(T^{n+1} x, T^{n+1} y\right) \leq M[d(x, y)$ $\left.+\cdots+d\left(T^{n} x, T^{n} y\right)\right]$. Since both $x$ and $y$ are fixed points, each summand on both sides is $d(x, y)$, and the inequality reduces to $n d(x, y) \leq \operatorname{Mnd}(x, y)<\operatorname{nd}(x, y)$. This is impossible unless $x=y$.

We conclude by presenting an example for which the standard Banach Contraction Principle is inapplicable, but for which Theorem 6.2 demonstrates the existence of a fixed point.

Example 6.3 Let $X$ be the closed interval [0,1/2] with the usual metric. Define $T x=x^{2}$. Then the hypotheses of Theorem 6.2 hold, but $\mathrm{T}$ is not a strict contraction in the sense of the Banach Contraction Principle.

Proof: Note first that $T^{2} x=x^{4}$. We assume WLOG 
that $x<y$ in the rest of the proof.

$$
\begin{aligned}
d(T x, T y) & =y^{2}-x^{2}=(y-x)(y+x) \\
& =d(x, y)(y+x)<d(x, y),
\end{aligned}
$$

since $x+y<1$.

Note that $\frac{d(T x, T y)}{d(x, y)}=\frac{y^{2}-x^{2}}{y-x}=y+x$. Since $y$ and $x$ can be chosen so that $y+x$ is arbitrarily close to 1 , there cannot be a constant $M$ with $0<M<1$ and $d(T x, T y) \leq M d(x, y)$. So the standard Banach Contraction Principle does not apply to this example.

However, we can show that there exists a constant $M$ with $0<M<1$ such that

$$
\begin{aligned}
d\left(T^{2} x, T^{2} y\right)+d(T x, T y) \leq & M[d(T x, T y)+d(x, y)] . \\
d\left(T^{2} x, T^{2} y\right)+d(T x, T y) & =y^{4}-x^{4}+y^{2}-x^{2} \\
& =\left(y^{2}+x^{2}\right)(y+x)(y-x) \\
& +(y+x)(y-x) \\
& =\left(y^{2}+x^{2}+1\right)(y+x)(y-x)
\end{aligned}
$$

$$
\begin{aligned}
d(T x, T y)+d(x, y) & =y^{2}-x^{2}+y-x \\
& =(y+x)(y-x)+y-x \\
& =(y+x+1)(y-x) .
\end{aligned}
$$

So the question of the existence of $M$ reduces, on division by $(y-x)$, to whether it is possible to find $M$ with $0<M<1$ and $\left(y^{2}+x^{2}+1\right)+(y+x) \leq M(y+x+1)$. The constant $M=0.8$ satisfies this inequality, because $1>y+x$ and $0.5>y^{2}+x^{2}$. So $0.8(y+x+1)>$ $1.6(y+x)>1.5(y+x)>\left(y^{2}+x^{2}+1\right)(y+x)$, completing the proof.

The authors would like to thank Prof. Jacek Jachymski, Mr. Merrick Sterling, and the referee for suggestions related to this paper.

\section{References}

[1] J. R. Jachymski, Schroder, Bernd; Stein, D., James Jr., “A connection between fixed-point theorems and tiling problems," Journal of Combinatorial Theory, Series A, Vol. 87, No. 2, 1999, pp. 273-286. doi:10.1006/jcta.1998.2960

[2] W. Rudin, "Principles of Mathematical Analysis," McGraw-Hill, New York, 1964. 